\title{
Telangiectatic osteosarcoma: A rare case in a 3-year-old female
}

Jiwei $\mathrm{Li}^{1}$, Liping Zhang ${ }^{2}$, Meifen Wang ${ }^{3}$, yucheng xie ${ }^{4}$, and changsha $\mathrm{wu}^{5}$

${ }^{1}$ Kunming Medical University

${ }^{2}$ Yunnan Provincial Hospital of Traditional Chinese Medicine

${ }^{3}$ Kunming Children's Hospital

${ }^{4}$ Kunming Children's Hospital

${ }^{5}$ Zhenxiong People's Hospital

July 21, 2020

\begin{abstract}
Telangiectatic osteosarcoma(TOS) is one of the rare subtypes of osteosarcoma(OS) and mainly affects the male of adolescents and young adults in the appendicular skeleton. We report a case of a 3-year-old girl who was diagnosed with TOS arising from the upper and proximal of the left humerus.
\end{abstract}

\section{Hosted file}

TOS (brief) . doc available at https://authorea.com/users/332317/articles/471119-telangiectaticosteosarcoma-a-rare-case-in-a-3-year-old-female 


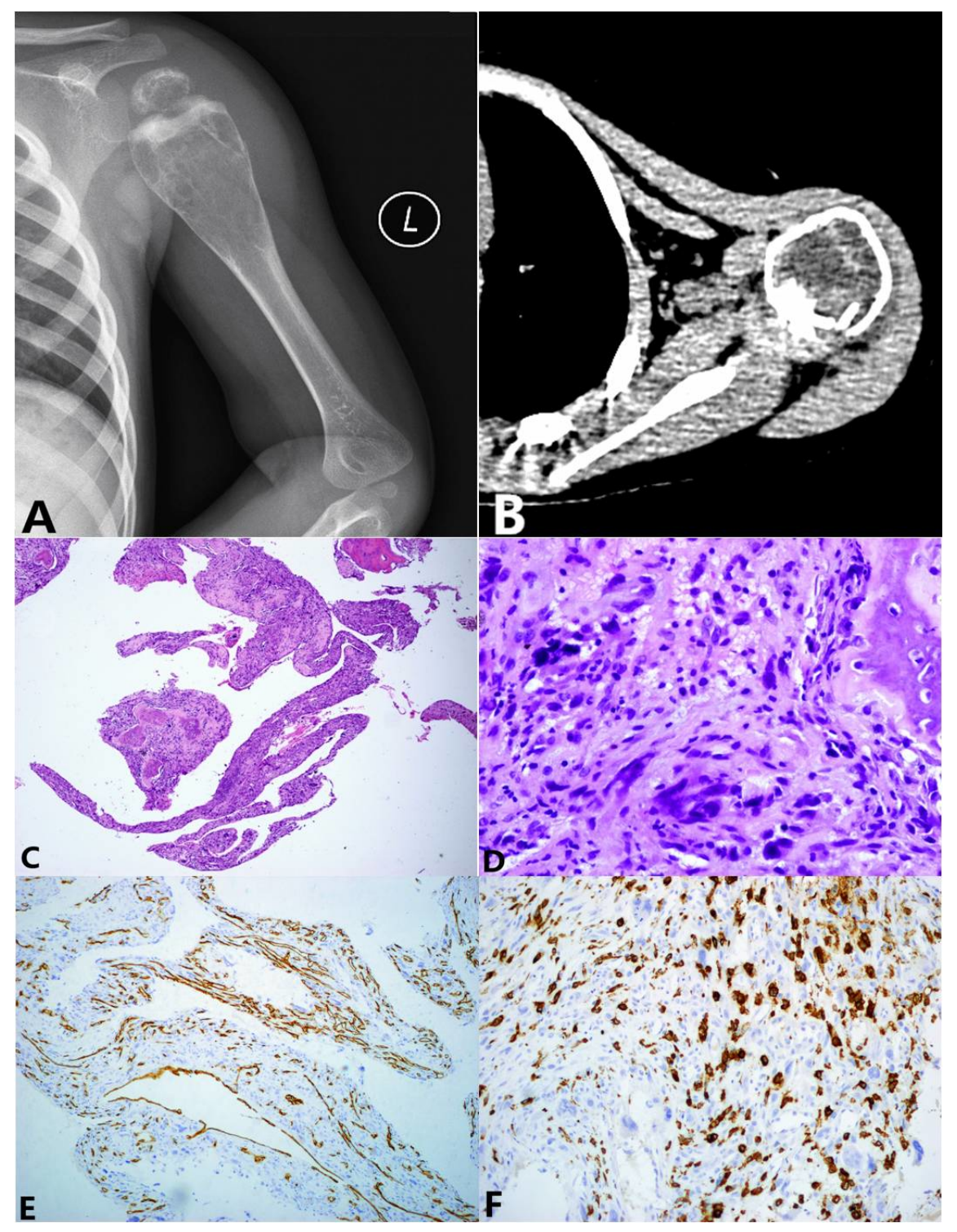

